\title{
THE INFLUENCE OF INHOMOGENEITY PRESENT IN LAVERED BIOLOGICAL STRUCTURE ON ELECTROMAGNETIC FIELD DISTRIBUTION
}

The knowledge of electrical properties of biological materials and their interaction with electromagnetic waves is necessary for suitable determining the effect of electromagnetic field on biological systems and, sequentially, prevention of undesirable consequences on human organism. Because of complex heterogeneous structure of human body the electromagnetic field distribution in biological systems is very complicated and depends, among others, on the type of tissue and on the presence of complicated layered structures and interfaces. With the knowledge of these electrical properties the absorption of energy and the field distribution which are the results of the solution to a boundary value problem can be obtained. This paper is focused on description of influence of inhomogeneity occurring in the human structure on electromagnetic field propagation. The inhomogeneity is represented by silicon brick and brain tumor placed into the model.

\section{Introduction}

Biological tissue is a complex and highly heterogeneous material. The dielectric properties of biological tissues have been of interest for over a century because they determine the pathways of current flow through the body and, thus, are very important in the analysis of wide range biomedical applications. The results from simple models phantoms of biological tissue for microwave hyperthermia at cancer therapy are not representative of the reality of different tissues, their associated shapes and boundaries, which will result in the electromagnetic (EM) propagation and power deposition rate. The reflected energy will be influenced not only by the wave impedance of each layer, but by the layer thickness as well. In a finite layered dielectric, multiple reflections and transmissions of electromagnetic wave occur at each boundary. Boundaries between tissues with divergent dielectric properties may produce localized hot spots and cannot be ignored. Analytical solution of a layered planar structure is given in this paper. The main concern of this paper is in influence of the inhomogeneity presence in layered structure on resulting signal.

The dielectric permittivity $(\varepsilon)$ is a parameter which characterizes the interaction between the electric field and the bound charges in the material. Due to bound charges for lossy materials the permittivity is complex variable with both real $\left(\varepsilon^{\prime}\right)$ and imaginary $\left(\varepsilon^{\prime \prime}\right)$ components. The interaction between the electric field and the bound charges in the tissue results in an oscillatory motion of the bound charges. The part of the motion that is $90^{\circ}$ out of phase with the electric field is characterized by the real part of the permittivity $\left(\varepsilon^{\prime \prime}\right)$ and is described as a lossless interaction. But as the bound charges oscillate, they also heat up due to friction-like forces within the molecule and from molecules nearby. This motion component is in phase with the electric field and is represented by the imaginary part of the permittivity $\left(\varepsilon^{\prime \prime}\right)$. From the electrical engineering point of view, studying the bulk dielectric properties, mainly permittivity and conductivity, remains the most direct way of characterizing any substance [1], [2].

\section{Wave propagation in biological materials}

If material conductivity does not equal zero value a material is lossy. Biological tissues are lossy materials and this loss changes the way the wave interacts with the material and its propagation behavior. Power will be distributed in the lossy material as wave passes through it, thus causing loss to the propagating wave. If EM energy is cumulated in the material, the material will heat up.

To better understand how EM power is deposited in a lossy material, considering theory of waves, Ampere's law can be written in a form

$$
\nabla \times \overline{\boldsymbol{H}}=\left(\sigma+\omega \varepsilon^{\prime \prime}\right) \overline{\boldsymbol{E}}+j \omega \varepsilon^{\prime} \overline{\boldsymbol{E}}
$$

where $\overline{\boldsymbol{H}}$ represents magnetic field intensity (A/m), $\overline{\boldsymbol{E}}$ represents electric field intensity $(\mathrm{V} / \mathrm{m}), \sigma$ is conductivity of the material and $\omega$ is an angular frequency ( $\mathrm{rad} / \mathrm{s})$. The first term on the right-hand side of this equation represents the current that produces a loss (heat) in the material through movement of free charges and bound charges. The last term in (1) is the displacement current which represents the lossless portion of the oscillation of the bound charges [3].

\footnotetext{
* Katarina Istenikova

Department of Measurement and Applied Electrical Engineering, Faculty of Electrical Engineering, University of Zilina, Slovakia,

E-mail: istenikova@fel.uniza.sk
} 
The loss properties of material are usually expressed as a loss tangent

$$
\operatorname{tg} \delta=\frac{\varepsilon^{\prime \prime}}{\varepsilon^{\prime}}=\frac{\sigma}{\omega \varepsilon_{r}^{\prime} \varepsilon_{0}}
$$

where $\sigma$ is the electrical conductivity $(\mathrm{S} / \mathrm{m}), \varepsilon_{r}^{\prime}$ is the relative dielectric constant, $\varepsilon_{0}=8.854191 \times 10^{-12}(\mathrm{~F} / \mathrm{m})$ is the permittivity of free-space and $\omega$ is angular frequency of applied electromagnetic field.

\subsection{Relaxation processes in biological tissues}

The response of biological tissues at microwave EM field is determined by the electro-chemical characteristics of cells, cellular structure and the intra-cellular fluid too. The permittivity of biological tissues is determined by several important dispersion phenomena whose contributions are normally restricted to specific band.

The dielectric spectrum of biological tissue is characterized by three main relaxation regions $\alpha, \beta$, and $\gamma$ at low, medium and high frequencies (more than $100 \mathrm{MHz}$ ) and other minor dispersions between $\beta$ and $\gamma$ are reported as $\delta$-dispersion, connected with proteins bound water [4]. The low frequency $\alpha$-dispersion is associated with ionic diffusion processes at the site of the cellular membrane The $\beta$-dispersion, in the hundreds of kilohertz region, is mainly caused by the polarization of cellular membrane, and finally the $\gamma$ dispersion, in the gigahertz region, is caused due to the polarization of the water molecules.

In general, dispersion characteristics of a large class of biological materials can be represented by Cole-Cole equation

$$
\dot{\varepsilon}=\varepsilon_{\infty}+\frac{\varepsilon_{s}-\varepsilon_{\infty}}{1+(j \omega \tau)^{1-p}},
$$

where $\varepsilon_{\infty}$ and $\varepsilon_{s}$ are the relative permittivities of material at infinite and zero frequencies respectively. The $(1-p)$ parameter represents distribution of relaxation in investigated material where $p$ is a distribution parameter in the interval $0 \leq \mathrm{p}<1$ [4].

The classical representation of frequency dependence of complex permittivity real and imaginary parts respectively to the diagram is in plotting the normalized real and imaginary part of the complex

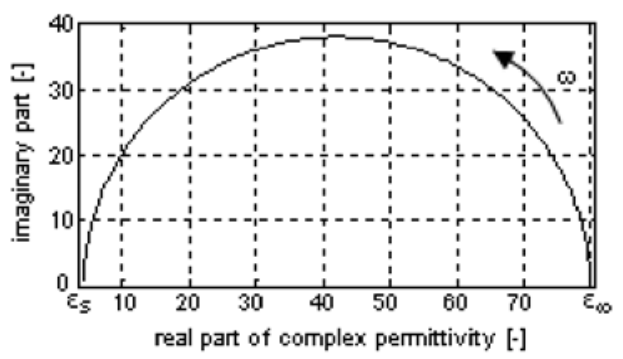

Fig. 1 Diagram for Cole-Cole equation in complex plane permittivity as functions of normalized frequency $f_{n}=\omega \tau$. A dependence of normalized relative permittivity and imaginary part of complex permittivity on normalized frequency calculated for pure water (a fundamental component occurred in biological tissues) is in Fig. 1. The main impact on the dielectric processes in investigated materials have frequencies in the region of dielectric dispersion where the relative permittivity decreases.

One of the most commonly used models represents the parts of complex permittivity plotted as functions of $\log _{10} \omega \tau$. In this case, these two parameters are presented independently of each other, Fig. 2.

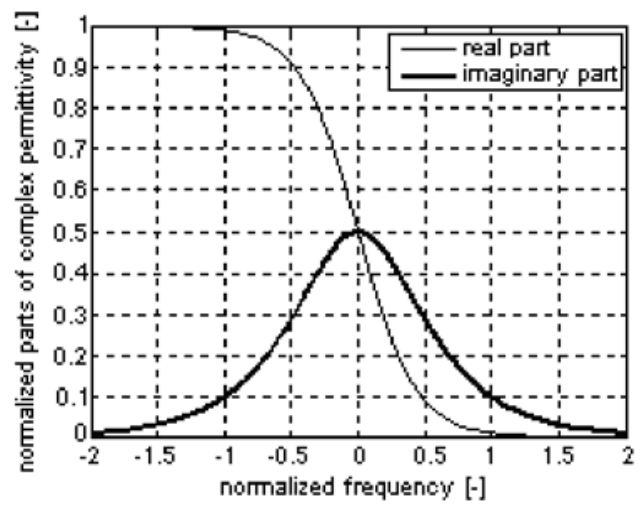

Fig. 2 Diagram for Cole-Cole equation, permittivity is a function of $\log _{10} \omega \tau$

Each biological material has its typical Cole-Cole diagram, which characterizes relaxation processes in chosen frequency region. Knowing the frequency dependence of real and imaginary part of complex permittivity enables the calculation of energy distribution in investigated biological tissue and optimization at hyperthermia planning at cancer treatment.

\subsection{Boundary conditions for biological materials}

Boundary conditions are very useful in interpreting and explaining characteristic behaviors of EM field interactions with biological systems. Because $\bar{E}$ is a vector, it can be resolved into two components, one tangential to the boundary and one normal to the boundary. Maxwell's equations require that the normal components of the $\bar{E}$ field at a charge-free boundary of two biological structures comply (4)

$$
\varepsilon_{1} E_{n 1}=\varepsilon_{2} E_{n 2},
$$

where $E_{n 1}$ is the normal component of the $\bar{E}$ field in structure 1 at the boundary, $E_{n 2}$ is the normal component of the $\bar{E}$ field in structure 2 at the boundary, $\varepsilon_{1}$ is the permittivity of structure 1 and $\varepsilon_{2}$ is the permittivity of structure 2 . 
The boundary condition for the tangential components of the field at the boundary of two structures is

$$
E_{t 1}=E_{t 2},
$$

where $E_{t 1}$ and $E_{t 2}$ are the tangential components of $\overline{\boldsymbol{E}}$ field at the boundary in structures 1 and 2, respectively [5]

Boundary conditions can give us a rough understanding of processes in every layers of biological sample. Fig. 3 shows that the tangential component of $\bar{E}$ field will pass through all layers of tissue without changing, as is indicated in (4). On the other hand, Fig. 4 shows that normal component of $\bar{E}$ field changed by the layers of tissue, as is given in (5). The field would be attenuated by the lossy tissues and reflections would occur within the layers.

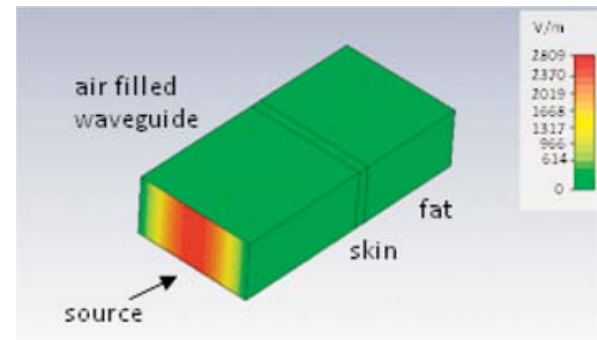

Fig. 3 The propagation of tangential component of the $\boldsymbol{E}$ field in skin - fat layered structure for mode $T E_{10}$

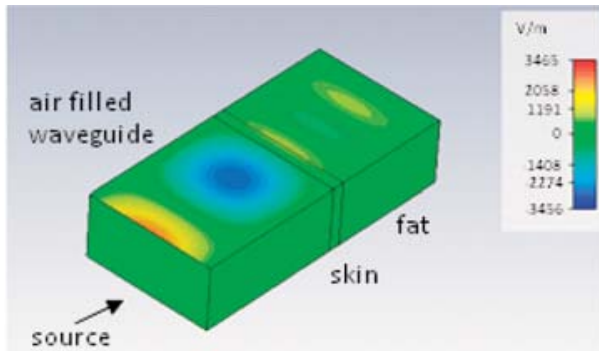

Fig. 4 The propagation of normal component of the $\boldsymbol{E}$ field in skin - fat layered structure for mode $T E_{10}$

\subsection{Reflection and transmission characteristics in layered structures}

When there are several layers of different tissues, the reflection and transmission characteristics become more complicated. Multiple reflections occur between tissue boundaries, with a resulting modification of the reflection and transmission coefficients, Fig. 5. The transmitted wave perpendicular to the boundary interacts with the reflected wave and both form standing waves in each layer. This phenomenon becomes especially pronounced if the thickness of each layer is less than the penetration depth for that tissue.

The un-dashed coefficients represent incident signal arriving from the left and the dashed ones represent incident signal arriv-

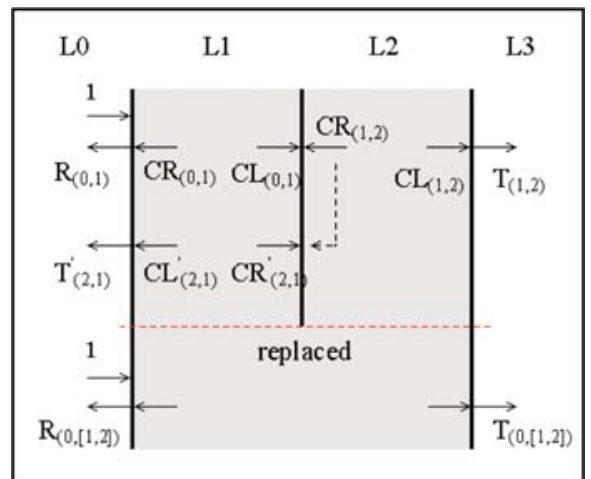

Fig. 5 Two-layered model with corresponding coefficients. $C R$ and $C L$ represent total sum of multiple reflections within sample

ing from the right. Then we can determine reflection and transmission coefficients

$$
\begin{aligned}
R_{(0,[1,2])} & =R_{(0,1)}+\frac{C L_{(0,1)} C R_{(1,2)} T_{(2,1)}}{1-C R_{(2,1)}^{\prime} C R_{(2,1)}}, \\
T_{(0,[1,2])} & =\frac{C L_{(0,1)} T_{(1,2)}}{1-C R_{(1,2)} C R_{(2,1)}^{\prime}} .
\end{aligned}
$$

This procedure can be then applied for $n$-layered structure [6].

\section{Numerical results}

To depict the behavior of the EM field within a layered biological sample without inhomogeneity, the model of two homogenous tissue layers was processed in the software environment CST Microwave Studio for electromagnetic simulation of high frequency components. For simulations the frequency $9.295 \mathrm{GHz}$ which comes under the X-band frequency range was used. The dielectric parameters used for different layers of biological sample were chosen according to our previous measurement results using Hippel's method [7]. These data are presented in Tab. 1.

Dielectric parameters of biological layers

Table 1

\begin{tabular}{|c|c|c|c|}
\hline & $\begin{array}{c}\text { Frequency } \\
f(\mathrm{GHz})\end{array}$ & $\begin{array}{c}\text { Permittivity } \\
\varepsilon^{\prime}(-)\end{array}$ & $\begin{array}{c}\text { Conductivity } \\
\sigma(\mathrm{S} / \mathrm{m})\end{array}$ \\
\hline skin & 9.295 & 41 & 0.15 \\
\hline fat & 9.295 & 5 & 0.04 \\
\hline water & 9.295 & 81 & 0.01 \\
\hline
\end{tabular}

Numerical results were performed for layered structure created from the skin layer with constant thickness $d_{1}=2 \mathrm{~mm}$ and the fat layer with thickness $d_{2}=15 \mathrm{~mm}$. Simulated layered structure was inserted into the waveguide with dimensions corresponding to the frequencies from $\mathrm{X}$ band.

In this case, the reflection coefficient of EM wave is influenced by energy storage in investigated biological material. The 
influence of the discontinuity presence on power loss density is in Fig. 6.

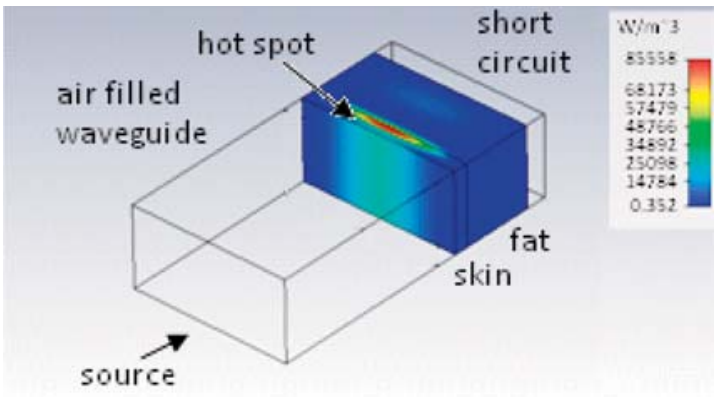

Fig. 6 The distribution of power loss density in skin - fat layered structure (short circuited wavegude)

A standing wave can be set up in the fat layer, which can create the raise of the energy storage. The EM fields with high density of electric field in the fat can create very painful subdermal hot spots, Fig. 6, that are difficult to treat, and must be avoided. A common way of mitigating this effect is to place a layer of water between the electromagnetic source and the body, Fig. 7. By propagating the field into the water first, the concentration of power at the surface of the body can be minimized and more uniform field distributions result. The water can be cooled to reduce the heat at and near the body surface.

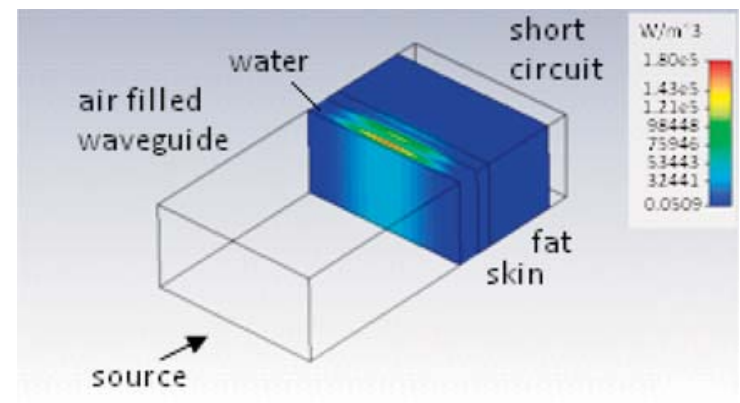

Fig. 7 The distribution of power loss density in skin - fat layered structure after placing the water layer between EM source and the body

To find out the influence of inhomogeneity presence in the volume of fat layer on electric energy field density distribution in layered structure was the main idea of this paper. The inhomogeneity - silicon brick - with dimensions $5 \times 2 \times 3 \mathrm{~mm}$, with relative permittivity $\varepsilon^{\prime}=11.9$ and the loss tangent $\operatorname{tg} \delta=0.004$ was inserted into the fat volume directly under the skin layer. The electric energy density in layered structure distribution with silicon is shown in Fig. 8.

From Fig. 8 it can be seen the strong influence of inhomogeneity presence in the volume of fat layer on EM field distribution in investigated biological layered structure. Fig. 8 allows us to determine location of areas in the volume of fat layer with the

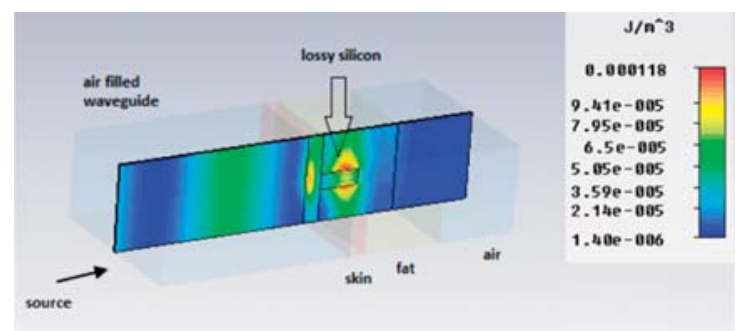

Fig. 8 The distribution of electric energy density in skin - fat layered structure (open waveguide) with silicon inhomogeneity.

spots characterised by higher electric field density. The temperature of biological material irradiated with high frequency EM field may arise in these areas.

The idea of next simulation was to find out the influence of tumor characterized with higher water content [2] presence on electric field energy density distribution and power loss distribution in layered biological structure. For this simulation a four-layered human head model consisting of skin, fat, bone and brain layer was created. The distance between the source and the media was choosen $4.5 \mathrm{~mm}$. Dielectric properties of each layer are presented in Tab. 2.

Dielectric properties of head tissues [5]

Table 2

\begin{tabular}{|c|c|c|c|}
\hline & $\begin{array}{c}\text { Thickness } \\
d(\mathrm{~mm})\end{array}$ & $\begin{array}{c}\text { Permittivity } \\
\varepsilon^{\prime}(-)\end{array}$ & $\begin{array}{c}\text { Conductivity } \\
\sigma(\mathrm{S} / \mathrm{m})\end{array}$ \\
\hline skin & 1.5 & 41 & 0.7 \\
\hline fat & 1 & 5 & 0.04 \\
\hline bone & 3 & 12 & 0.95 \\
\hline
\end{tabular}

The inhomogeneity - spherical tumor - with diameter of $3 \mathrm{~mm}$ and with relative permittivity $\varepsilon^{\prime}=38$ and conductivity $\sigma=11 \mathrm{Sm}^{-1}$ was inserted into the brain layer under the bone layer. This inhomogeneity is different in size and shapes in comparison with surrounding tissues. The electric energy density and power loss density in layered structure distribution with tumor is shown in Figs. 9 and 10 .

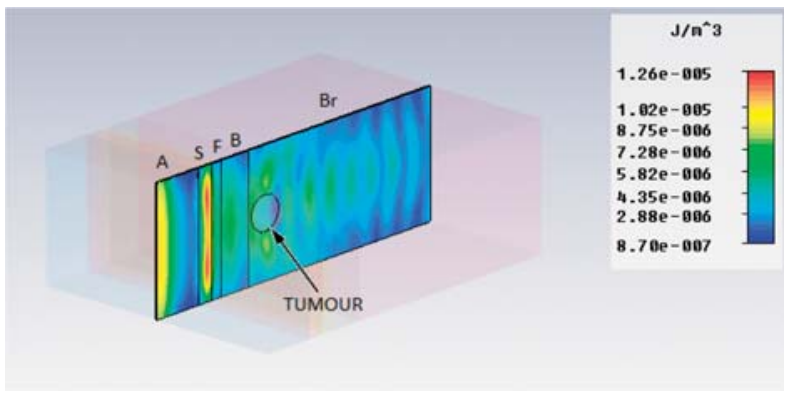

Fig. 9 The distribution of electric energy density in the multilayered human head model with presence of brain tumor, $A$ - air, $S$ - skin, $F$ - fat, $B$ - bone, $B r$ - brain 


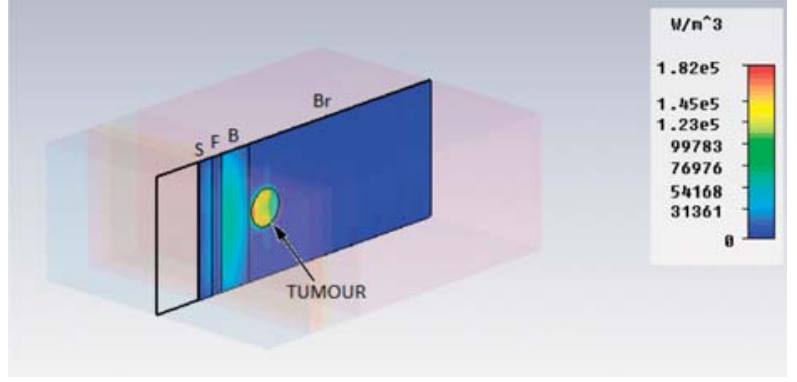

Fig. 10. The distribution of power loss density in the multilayered human head model with presence of brain tumor, $A$ - air, $S$ - skin, $F$ - fat, $B$ - bone, $B r$ - brain

In the figures it is shown that the presence of tumor in the brain has a high influence on EM field distribution with result of having more field concentration than the surrounding brain layer.

\section{Conclusion}

Because of huge introduction of equipment which emit EM fields of various frequency ranges and intensities the scientists in electrical engineering are focused to study the way of EM field interaction with biological tissues. The experimental and numerical results connected with the study of dielectric properties of biological materials and their properties in the electromagnetic field and, on the other hand, the influence of dielectric properties of biological material on EM wave propagation gives us the information useful in medical diagnostics and therapy using microwaves. The microwave dielectric method has been one of the most reliable techniques for investigation of the dynamic structure of macromolecules and dielectric relaxation measurements have been applied to the investigation of dielectric properties of various biological tissues. This and other similar information inspired us to pay attention to observation of some materials behavior in biological tissues, particularly in tumors and layered structures.

The presented numerical results are useful at the microwave generator parameters optimization used for microwave hyperthermia at malignant tumors treatment. The obtained numerical results can be also useful at design of chosen human body part phantoms. In the view of this analytical approach which allows us to evaluate coefficients characterizing propagation of EM wave in the layered structure, the next parameters of EM wave like SAR (specific absorption rate) or the presence of inhomogeneities with different dielectric characteristic in investigated structure can be calculated [8]. Further theoretical and experimental investigations will be carried out to temperature dependences of dielectric properties of biological tissues, because it is inevitable to investigate the penetration of microwaves through different layers in clinical practice using microwaves.

\section{Acknowledgment}

The work has been done in the framework of Grant VEGA 1/0761/08 „Design of Microwave Methods for Materials Nondestructive Testing" of the Ministry of Education of the Slovak Republic and project APVV-0535-07.

\section{References}

[1] TOFIGHI, M.R., DARYOUSH, A.: Measurement Techniques for the Electromagnetic Characterization of Biological Materials, Handbook of Engineering Electromagnetics, CRC Press, 2004.

[2] PEYMAN. A.: Dielectric Properties of Tissues, Variation with Structure and Composition, Proc. Conference ICAA, 2004, 863-864.

[3] FURSE C., CHRISTENSEN D. A., DURNEY C. H.: Electric and Magnetic Fields: Basic Concepts, Basic Introduction to Bioelectromagnetics, CRC Press, 2009.

[4] CHRIST, A., KLINGENBOCK, A., SAMARAS, T., GOICEANU, C., KUSTER, N.: The Dependence of Electromagnetic Far-field Absorption on Body Tissue Composition in the Frequency Range from $300 \mathrm{MHz}$ to $6 \mathrm{GHz}$, IEEE Transaction on Microwave Theory and Techniques, vol. 54, 2006, 2188-2195.

[5] AKRAM, G., JASMY, Y.: LabVIEW-Based Planar Multilayered Model for Estimation of the Absorbed Energy Inside Biological Tissues, IEEE Antennas and Propagation Magazine, vol. 50 (2), 2008, 152-158.

[6] ZRNIK, J.: Numerical Modelling of Electromagnetic Wave Interaction with Heterogeneous Biological Structure, Proceedings of AMTEE'09, 2009, VI-13 - VI-14.

[7] ISTENIKOVA, K., FAKTOROVA, D.: Influence of Biological Materials Dielectric Parameters on Electromagnetic Wave Propagation, Proceedings of Elektro'10, 2010, TA5_44 - TA5_48.

[8] ISTENIKOVA, K., FAKTOROVA, D.: Modelling of Scattering Parameters in Biological Tissues, Proceedings of CPEE'10, p. 2010. 